\title{
Intraductal Papillary Mucinous Neoplasm (IPMN) of the pancreas: a case study and review of literature
}

\author{
Junaid Nabi ${ }^{1}$, Fatema N Authoy ${ }^{1}$, Ghulam N Sofi ${ }^{2}$ \\ ${ }^{I}$ Department of General Surgery, Shaheed Suhrawardy Medical College Hospital, Bangladesh \\ ${ }^{2}$ Department of Pathology, Sher-e-Kashmir Institute of Medical Sciences (SKIMS), India
}

\begin{abstract}
Intraductal papillary mucinous neoplasm (IPMN) of the pancreas is one of the two entities which come under the realm of non-inflammatory cystic lesions of the pancreas, the other one being mucinous cystic neoplasm. The clinco-pathological features of intraductal papillary mucinous neoplasm of the pancreas are unique. The patient had a long history of symptoms suggestive of chronic pancreatitis. Endoscopic retrograde cholangiopancreatography revealed dilatation of the main duct, ultrasonography showed cystic dilatation interpreted as pseudocyts. Current thinking suggests that at best IPMN represents a premalignant condition and, as such, surgical intervention is recommended. The aim should be to resect all gross disease while attempting to achieve a negative surgical margin, which in the majority of cases can be achieved by a partial or subtotal pancreatectomy, which was performed on the patient. Histologically, they demonstrate a spectrum of cellular atypia ranging from minimal mucinous hyperplasia to frank invasive carcinoma. Although the neoplasms are less aggressive as a group than conventional pancreatic ductal adenocarcinoma, patients with IPMNs may pursue a deadly course, even in the absence of identifiable invasive carcinoma thus emphasizing the importance of recognizing IPMNs and initiating optimal modalities of treatment.
\end{abstract}

Keywords: Intraductal Papillary Mucinous Neoplasm (IPMN), pancreatic neoplasm, partial pancreatectomy

\section{Introduction}

Intraductal papillary mucinous neoplasm (IPMN) of the pancreas is a relatively recently described entity, first documented in 1982 [1]. It has been the subject of several clinicopathological, radiological, and molecular analyses. IPMN is defined as grossly visible, non-invasive, mucin producing, predominantly papillary epithelial neoplasms arising from the main pancreatic duct or branch ducts, and associated with variable distension of the associated duct [2]. In addition, varying grades of cytological atypia may be encountered in the lining epithelium. Usually IPMNs are not associated with inherited syndromes and most cases occur sporadically.

In 1989, Morohoshi et al. [3] reported series of Intraductal Papillary Neoplasm (IPN) of the pancreas for the first time, other similar tumors have been reported in the literature with different names such as Intaductal papilloma, CA in-situ of the pancreas, diffuse villous Carcinoma of the duct of Warsung.

During the past 15 years, intraductal papillary mucinous neoplasms have been reported with increasing frequency, representing an estimated $0.5-10 \%$ of pancreatic exocrine neoplasms [4]. This supports the hypothesis of progression from IPMN adenoma to carcinoma through borderline neoplasms. It is unknown whether all IPMNs have potential malignancy, and also what timescale is required for neoplastic progression to invasive carcinoma [5]. The report contributes to the currently active discussion of the group of neoplasms in the area of pancreatic pathology and management of such cases.

\section{Clinical Summary And Methods}

The patient was a 64-year-old man, who presented with recurrent episodes of epigastric pain which was moderate to severe in intensity. After taking a through history and physical examination a diagnosis of chronic pancreatitis was made. Treatment history revealed that earlier a cholecystectomy was done for cholelithiasis. When investigations were ordered, serum amylase levels were elevated and ultrasonography showed cysts in the pancreas (pseudocysts). Endoscopic retrograde cholangiopancreatography (ERCP) showed dilatation of the main duct. A partial or subtotal pancreatectomy was performed.

On gross examination of the resected specimen, pancreas was enlarged; firm with nodularity but no mass was seen. To access the histopathology, the specimen was received in a $10 \%$ neutral buffered formalin. The entire pancreatic specimen was processed for histopathological evaluation. After fixation, multiple serial sections were taken (5mm thickness)-paraffin embedded, cut vertically into 10-15 serial sections processed in a routine manner and stained with haematoxylin and eosin.

The microscopic examination revealed a lesion in a branch duct within the pancreas which was papillary in configuration, causing distension of the duct, and was surrounded by sclerotic fibrous tissue, Extracellular mucin production was present with resultant distension of the duct housing the lesion. Moderate to 
severe dysplastic changes of lining epithelium was seen and mitosis was not seen (Figure-1). No stromal infiltration was seen, a diagnosis of IPMN with mild to moderate dysplasia was made. The surrounding pancreatic tissue showed features of chronic pancreatitis secondary to duct obstruction. No lymph node metastases were seen.

\section{Discussion}

Intraductal papillary mucinous neoplasm is a rare pancreatic tumor with characteristic features of papillary structures, moderate to severe dysplastic changes, piling of epithelium with cystically dilated ducts filled with mucus. No stromal infiltration was seen, age and sex distribution, clinical profile of chronic pancreatitis. Our study is in accordance with larger series of IPMNs as reported by Morohoshi et al. [3].

Before the term IPMN was proposed to encompass the spectrum of these tumors under one heading, they had been reported in the literature under a plethora of terms, including villous adenomas or intraductal papillary neoplasms, mucin-producing tumors or intraductal mucin-hypersecreting tumors, and mucinous duct ectasia or ductectatic mucinous cystic neoplasms [6]. Our study confirms that there is significant histologic overlap among these clinical entities and that it is justified to categorize them as one group: IPMN. This unifying category has found broad acceptance and is endorsed by the WHO [7]. This study gives further information of clinical history of chronic pancreatitis- confirmed both clinically and histologically with presumption that chronic pancreatitis is a precancerous condition [8].

Understanding of IPMNs has increased substantially since 2000, when Irie et al. reported a series of radiological parameters that could indicate degeneration [9]. The study was interesting for two reasons: the first being that the mean age of these patients is rather high, between sixth and seventh decade, with a consequent risk of co-morbidity, while the second was related to the necessity of extensive pancreatic surgery for a neoplasm with a slow evolution. Nonetheless, it is uncertain whether all IPMNs have a malignant evolution or whether the progression of these tumors is so slow that it represents a secondary problem in an elderly population. This seems particularly true for branch duct (BD) IPMNs $<2 \mathrm{~cm}$ in diameter without solid elements, in which the prevalence of diagnosis has being increasing due to what could be termed a disease of technology [10].

\section{Conclusion}

All of these clinic-pathological findings suggest chronic pancreatitis has a definite role in the genesis of the tumor. Chronic panceatitis leads to ductal hyperplasia, proliferative changes, cellular pleomorphism progressing to in-situ carcinomatous changes. Although gross and cytologic findings can aid in confirming the suspected diagnosis, an integration of complete clinical, sonographic, and cytologic information may be the best way to reach the most appropriate diagnosis possible. Our review of the literature indicates that preoperative indicators of malignancy in IPMN are still lacking. Concerning resection margins, complete tumor resection is usually possible by partial pancreatectomy. Malignant relapses are not uncommon. Incomplete resection and diffuse or multifocal tumor represent poor prognostic factors. Total pancreatectomy should be considered in such cases. These neoplasms may be less aggressive than pancreatic ductal adenocarcinoma, but patients with IPMNs may pursue a deadly course, even in the absence of identifiable invasive carcinoma, thus emphasizing the need of early recognition IPMN.

\section{References}

[1] Ohhashi K, Murakami M, Murayama M, et al. Four cases of mucous secreting pancreatic cancer. Prog Dig Endosc 1982; 20:348-51.

[2] Klimstra DS, Takaori K, Hruban RH, et al. Consensus criteria for the classification of pancreatic intraepithelial neoplasia (PanIN) and intraductal papillary mucinous neoplasms (IPMNs). Mod Pathol 2004; 17:305A.

[3] Morohoshi T, Kanda M, Sanduma K, Kloppel G. Intraductal Papillary Neoplasms of the pancreas: clinic-pathological study of six patients. Cancer 1989; 64:1329-35

[4] Falconi M, Salvia R, Bassi C, et al. Clinicopathological features and treatment of intraductal papillary mucinous tumour of the pancreas. Br J Surg 2001; $88: 376-81$.

[5] Fujiwara T, Tu Y, et al. Long-term follow-up of intraductal papillary adenoma of the pancreas. J Gastroenterol 2002; 37:868-73.

[6] Itai Y, Ohhashi K, Nagai H, Murakami Y, Kokubo T, Makita K, et al. "Ductectatic" mucinous cystadenoma and cystadenocarcinoma of the pancreas. Radiology 1986; 161:697-700.

[7] Kloppel G, Solcia E, Longnecker DS, Capella C, Sobin LH, editors. Histologic typing of tumors of the exocrine pancreas (Geneva: Springer, 1996).

[8] Kozuka S, Sassa R, Taki J, et al. Relation of pancreatic duct hyperplasia to carcinoma. Cancer 1979; 43:1418-28.

[9] Irie H, Honda H, Aibe H, et al. MR cholangiopancreatographic differentiation of benign and malignant intraductal mucin-producing tumors of the pancreas. Am J Roentgenol 2000; 174:1403-8.

[10] Krinsky G. Case 26-2000: intraductal papillary mucinous carcinoma. N Engl J Med 2001; 344: 141; author reply 141-2. 


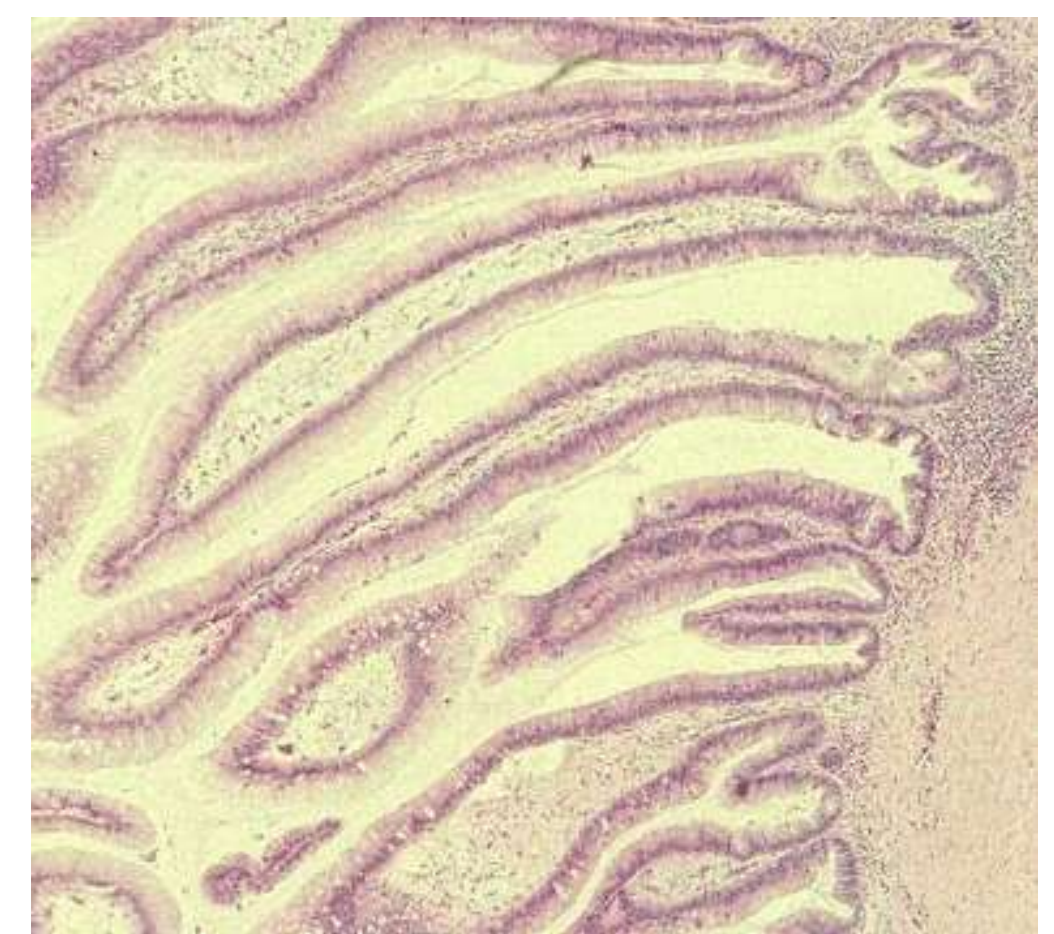

Figure 1: 64-year-old man with intraductal papillary mucinous neoplasm of pancreas.

Microphotograph of specimen showing tumor composed of papillary structures with piling epithelium and dysplastic changes to the epithelium (H\&E). 